05,12

\title{
Ферромагнитный резонанс в монокристаллических спиновых вентилях CoFeB/Ta/CoFeB и пленках CoFeB с перпендикулярной магнитной анизотропией
}

\author{
(С) А.Д. Таланцев ${ }^{1}$, Г.Л. Львова ${ }^{1}$, O.В. Коплак ${ }^{1,2}$, O.C. Дмитриев ${ }^{3}$, Y. Lu $^{4}$, \\ S. Mangin ${ }^{4}$, P.Б. Моргунов 1,3, ฯ \\ ${ }^{1}$ Институт проблем химической ффизики РАН, \\ Черноголовка, Россия \\ ${ }^{2}$ Балтийский фредеральный университет им. И. Канта, \\ Калининград, Россия \\ ${ }^{3}$ Тамбовский государственный технический университет, \\ Тамбов, Россия \\ ${ }^{4}$ Institut Jean Lamour, UMR 7198 CNRS, Université de Lorraine, \\ Nancy, France \\ ๑ E-mail: morgunov2005@yandex.ru
}

(Поступила в Редакцию 21 февраля 2017 г.)

В пленке $\mathrm{MgO} / \mathrm{CoFeB} / \mathrm{MgO} / \mathrm{Ta}$ с одним ферромагнитным слоем (монослой) и спиновом вентиле $\mathrm{MgO} / \mathrm{CoFeB} / \mathrm{Ta} / \mathrm{CoFeB} / \mathrm{MgO} / \mathrm{Ta}$, состоящем из двух ферромагнитных слоев $\mathrm{CoFeB}$ с монокристаллической структурой, разделенных немагнитным слоем Та (бислой), исследованы ориентационные зависимости ферромагнитного резонанса. Анализ ориентационных зависимостей структур с перпендикулярной магнитной анизотропией позволил извлечь константы магнитной анизотропии, а также коэффициенты демпфирования. Обсуждаются физические причины различий этих параметров в однослойных и двухслойных структурах.

Работа поддержана Министерством образования и науки РФ (грант № 3.1992.2017/ПЧ в рамках конкурса научных проектов, выполняемых научными коллективами исследовательских центров и (или) научных лабораторий образовательных организаций высшего образования), а также грантом № 14.W03.31.0001.

DOI: 10.21883/FTT.2017.08.44753.47

\section{1. Введение}

Инженерия спиновых логических вентилей на основе туннельного гигантского магнетосопротивления (ГМС) успешно завоевывает свою нишу в индустрии элементов магнитной памяти, считывающих и записывающих устройств, биосенсоров [1-3]. Главным элементом таких устройств является гетероструктура с несколькими ферромагнитными слоями, разделенными немагнитными прослойками. Имеется большое количество модификаций таких гетероструктур, однако до сих пор инженерные, технологические и фундаментальные аспекты их функционирования до конца неясны. Среди „белых пятен“ можно упомянуть отсутствие систематической информации о роли доменных стенок и фазы обратной намагниченности при перемагничивании ферромагнитных слоев, нестабильность и сложность поведения намагниченности на рабочих частотах (более $10 \mathrm{GHz}$ ), неадекватность теории макроспина при описании релаксационных процессов в вентилях и многие другие. Даже самые простые гетероструктуры, состоящие из пары ферромагнитных слоев $\mathrm{CoFeB}$, популярные у исследователей, продолжают привлекать внимание в связи с многообразием физических эффектов, а также появлением новых идей их использования в спинтронике [4-8].

Настоящая работа посвящена исследованию и сравнению ферромагнитного резонанса (ФМР), возбуждаемого в отдельном слое $\mathrm{CoFeB}$, а также в двухслойной системе $\mathrm{CoFeB} / \mathrm{Ta} / \mathrm{CoFeB}$ в условиях существования перпендикулярной магнитной анизотропии ферромагнитных слоев. Перпендикулярная анизотропия в таких системах, как известно, обеспечивается гибридизацией $3 d$-орбиталей переходных металлов (Co,Fe) с $\mathrm{O}_{2 p}$-орбиталями оксида $\mathrm{MgO}$. Такая гибридизация обеспечивает перпендикулярную магнитную анизотропию на границе $\mathrm{CoFeB} / \mathrm{MgO}$ [9]. При толщинах слоев CoFeB менее $2 \mathrm{~nm}$ вклад перпендикулярной анизотропии начинает доминировать, и ось легкого намагничивания пленок оказывается направленной перпендикулярно плоскости слоев. Монокристаллические образцы, приготовленные нами, обладают ГМС в среднем до $100 \%[10,11]$ и могут рассматриваться в качестве готовых спиновых вентилей. ФМР, возбуждаемый в тонких пленках с кубической структурой, сам по себе хорошо известен и не представляет самостоятельного интереса. Однако сравнение ФМР в тонкой пленке и двухслойной системе показывает существенные различия, что является предметом обсуждения в настоящей работе.

Еще один важный параметр гетероструктур спинтроники - коэффициент затухания прецессии намагниченности $\alpha$, который является феноменологическим параметром в уравнении Ландау-Лифшица-Гильберта [1-3]. Именно этот параметр определяет пригодность или непригодность созданных гетероструктур для практиче- 
ского использования на сверхвысоких частотах. В инженерной практике принято, что верхний предел значений $\alpha$, приемлемый для использования, составляет 0.027 . Причина этого ограничения состоит в том, что перемагничивание вентилей в реальных устройствах производится с помощью спин-поляризованного тока, прямо пропорционального величине $\alpha$. При высоком значении величины $\alpha$ нужен значительный спин-поляризованный ток, чтобы развернуть вектор намагниченности слоя на $180^{\circ}$ и переключить спиновый вентиль. Это делает неприемлемыми структуры с большим значением $\alpha$. Поэтому еще одной целью настоящей работы является установление того, как меняется коэффициент затухания при переходе от однослойной к двухслойной системе.

\section{2. Образцы и методика экспериментов}

Многослойные структуры $\mathrm{MgO}(2.5 \mathrm{~nm}) /$ $\mathrm{CoFeB}(1.1 \mathrm{~nm}) / \mathrm{MgO}(2.5 \mathrm{~nm}) / \mathrm{Ta}(5 \mathrm{~nm})$ (образец I монослой с одним слоем $\mathrm{CoFeB})$ и $\mathrm{MgO}(2.5 \mathrm{~nm}) /$ $\mathrm{CoFeB}(1.1 \mathrm{~nm}) / \mathrm{Ta}(0.75 \mathrm{~nm}) / \mathrm{CoFeB}(0.8 \mathrm{~nm}) / \mathrm{MgO}(2.5 \mathrm{~nm}) /$ $\mathrm{Ta}(5 \mathrm{~nm})$ (образец II - бислой, состоящий из двух ферромагнитных слоев $\mathrm{CoFeB}$, разделенных антиферромагнитным слоем Та) были выращены на подложках нелегированного GaAs(001) методом магнетронного распыления. Подробно методика роста описана в работах $[10,11]$. Для увеличения перпендикулярной анизотропии производился быстрый температурный отжиг при $250^{\circ} \mathrm{C}$ в течение $3 \mathrm{~min}$, который приводил к увеличению кристалличности слоев $\mathrm{CoFeB}$. Характерный размер образцов составлял $0.4 \times 2 \times 2 \mathrm{~mm}$. Отметим, что толщина пленки $\mathrm{CoFeB}$ в монослойном образце точно совпадала с толщиной одной из пленок бислойного образца: $1.1 \mathrm{~nm}$. Такой подбор образцов был необходим для корректного сравнения магнитного резонанса в этих структурах, чтобы в случае обнаружения различий они не объяснялись тривиальным эффектом различия толщин пленок. Ферромагнитный резонанс был исследован с помощью ЭПР-спектрометра $X$-диапазона Bruker ESP 300. Частота микроволнового поля составляла $9447 \mathrm{~Hz}$, мощность $6.3 \mathrm{~mW}$, частота модулирующего поля $100 \mathrm{kHz}$, амплитуда модулирующего поля $10 \mathrm{Oe.}$

\section{3. Экспериментальные результаты и их обсуждение}

Анизотропные спектры ФМР для монослоя и бислоя, измеренные при температуре $296 \mathrm{~K}$ в четырех ориентациях магнитного поля относительно нормали к плоскости образца: $\theta=0,45,60$ и 90 , представлены на рис. $1, a, b$. В спектрах наблюдалась интенсивная несимметричная линия $A$, положение которой зависит от угла $\theta$, соответствующая сигналу от образца (рис. $1, b)$. Изотропная линия слабой интенсивности $B$ с центром на 2700 Ое соответствует сигналу контрольного образца. Далее обсуждается только линия $A$.
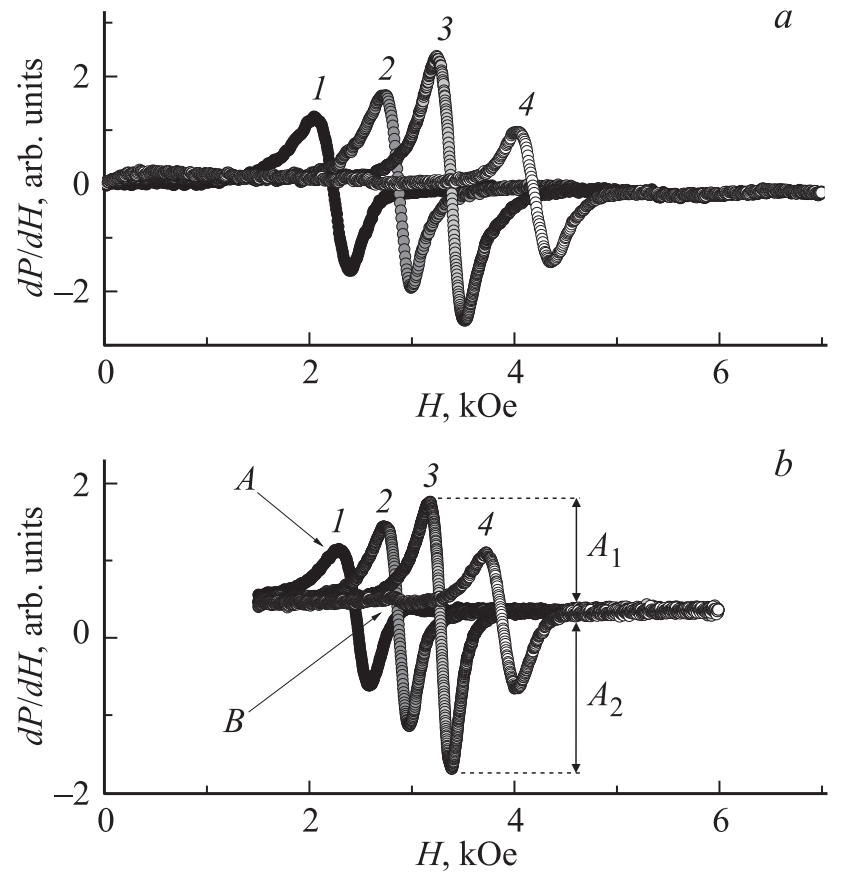

Рис. 1. Спектры ФМР монослойного $(a)$ и бислойного $(b)$ образцов для четырех ориентаций $\theta$ магнитного поля $H$ относительно нормали к плоскости образца: $\theta=0$ (1), 45 (2), 60 (3) и $90^{\circ}(4)$. Пик при 2850 Ое соответствует сигналу контрольного образца и не зависит от угла $\theta$.

Зависимость резонансного поля линии $H_{\mathrm{RES}}$ от угла как для монослоя, так и для бислоя имела классический вид и в интервале углов от -0 до $360^{\circ}$ содержала два максимума (при $\theta=90$ и $270^{\circ}$ ) и два минимума (при $\theta=0$ и $\left.180^{\circ}\right)$. При этом для монослоя разность резонансных полей при $\theta=0$ и 90 больше, чем разность резонансных полей для бислоя. Это означает, что энергия перпендикулярной анизотропии выше для монослойного образца. Причиной такого различия энергий анизотропии монослоя и бислоя может служить то, что перпендикулярная анизотропия — это поверхностная анизотропия. Она возникает вследствие гибридизации орбиталей Со и $\mathrm{O}_{2 p}$ на интерфейсе $\mathrm{CoFeB}$ и $\mathrm{MgO}$. Ее вклад будет тем более существенным, чем тоньше слой и чем больше площадь интерфейса между $\mathrm{CoFeB}$ и $\mathrm{MgO}$ на единицу объема слоя. В бислойном образце слои $\mathrm{CoFeB}$ граничат с разделяющим слоем Та с одной стороны и с $\mathrm{MgO}$ с другой. В монослойном образце $\mathrm{MgO}$ находится по обеим сторонам слоя $\mathrm{CoFeB}$, в результате чего площадь интерфейса $\mathrm{CoFeB} / \mathrm{MgO}$ для монослоя оказывается выше.

Константы анизотропии могут быть найдены с помощью стандартных аппроксимаций зависимости резонансного поля от полярного угла для магнитокристаллической анизотропии [12-15]. Зависимость резонансного поля ферромагнитной пленки кубической симметрии от полярного $\theta$ и азимутального $\varphi$ углов с учетом констант кристаллической анизотропии второго $K_{2 \perp}$, 
$K_{2 \|}$ и четвертого $K_{4}$ порядков следует из магнитной части свободной энергии, которая запишется в виде [12]

$$
\begin{aligned}
E= & -\mathbf{H M}-\left(K_{2 \perp}-2 \pi M^{2}\right) \cos ^{2} \theta \\
& +K_{2 \|} \sin ^{2} \theta \cos ^{2}\left(\varphi-\varphi_{2 \|}\right)+E_{4}\left(K_{4}, \theta, \varphi\right),
\end{aligned}
$$

где $M$ - намагниченность насыщения, а энергия четвертого порядка анизотропии равна $[13,14]$

$$
E_{4}=-\frac{1}{2} K_{4}\left[\cos ^{4} \theta+\frac{1}{4}(3+\cos 4 \varphi) \sin ^{4} \theta\right] .
$$

Резонансная угловая частота $\omega$ запишется в виде

$$
\begin{aligned}
\left(\frac{\omega}{\gamma}\right)^{2}= & \frac{1}{M^{2}}\left[E_{\theta \theta}\left(\frac{E_{\varphi \varphi}}{\sin ^{2} \theta}+\frac{\cos \theta}{\sin \theta} E_{\theta}\right)\right. \\
& \left.\times\left(\frac{E_{\theta \varphi}}{\sin \theta}-\frac{\cos \theta}{\sin \theta} \frac{E_{\varphi}}{\sin \theta}\right)^{2}\right],
\end{aligned}
$$

где $E_{\varphi \varphi}, E_{\theta \varphi}, E_{\theta}$ и $E_{\varphi}-$ производные от энергии (1) по соответствующим углам [15].

В результате дифференцирования соотношений (1) и (2) и подстановки в (3) получим условие для резонансного поля $H_{\mathrm{RES}}$

$$
\begin{aligned}
& \left(\omega_{0} / \gamma\right)^{2}=\left(H_{\mathrm{RES}} \cos \left(\theta-\theta_{H}\right)-4 \pi M_{\mathrm{eff}} \cos ^{2} \theta\right) \\
& \quad \times\left(H_{\mathrm{RES}} \cos \left(\theta-\theta_{H}\right)-4 \pi M_{\mathrm{eff}} \cos (2 \theta)+H_{2 \|}\right),
\end{aligned}
$$

в котором $4 \pi M_{\text {eff }}=\left(-2 K_{2 \perp} / M_{S}\right)+4 \pi M_{S}-$ эффективное поле, $\omega_{0}=9.447 \mathrm{GHz}$ - резонансная частота спектрометра, $\gamma=g / \mu_{\mathrm{B}} h-$ гиромагнитное отношение, $M_{S}$ - намагниченность насыщения слоя, $K_{2 \perp}-$ искомое значение константы анизотропии, $\theta-$ угол между вектором намагниченности и нормалью к плоскости образца, $\theta_{H}-$ угол между направлением магнитного поля и нормалью к плоскости образца.

Вклад анизотропии в плоскости образца (in-plane) пренебрежимо мал по сравнению с анизотропией относительно полярного угла (out-of-plane). Направление оси анизотропии определяется знаком эффективного поля $H_{\text {eff. }}$ Если $\left(-2 K_{2 \perp} / M_{S}\right)+4 \pi M_{S}>0$, ось легкого намагничивания лежит в плоскости пленки. Если же $\left(-2 K_{2 \perp} / M_{S}\right)+4 \pi M_{S}<0$, ось легкого намагничивания перпендикулярна плоскости пленки. В нашем случае $H_{\text {eff }}<0$ как для монослоя, так и для бислоя.

Результаты аппроксимации угловых зависимостей резонансного поля показаны на рис. 2, $a$ сплошными линиями. При аппроксимации были получены следующие параметры: для монослоя $M_{S}=775 \mathrm{G}, g=1.95$, $K_{2 \perp}=4.2 \cdot 10^{6} \mathrm{erg} / \mathrm{cm}^{3}, \quad K_{S}=K_{2 \perp} V / S=0.42 \mathrm{erg} / \mathrm{cm}^{2}$ $(V$ и $S-$ объем и площадь поверхности монослоя соответственно), $\quad 4 \pi M_{\mathrm{eff}}=1230 \mathrm{G}$; для бислоя $4 \pi M_{\text {eff }}=890 \mathrm{G}, g=2.025$. В спиновом вентиле в более тонком слое $\mathrm{CoFeB}$ толщиной $0.8 \mathrm{~nm}$ были получены следующие параметры: $M_{S}=657 \mathrm{G}$, $K_{2 \perp}=3.0 \cdot 10^{6} \mathrm{erg} / \mathrm{cm}^{3}, \quad K_{S 2}=0.3 \mathrm{erg} / \mathrm{cm}^{2} . \quad$ В более
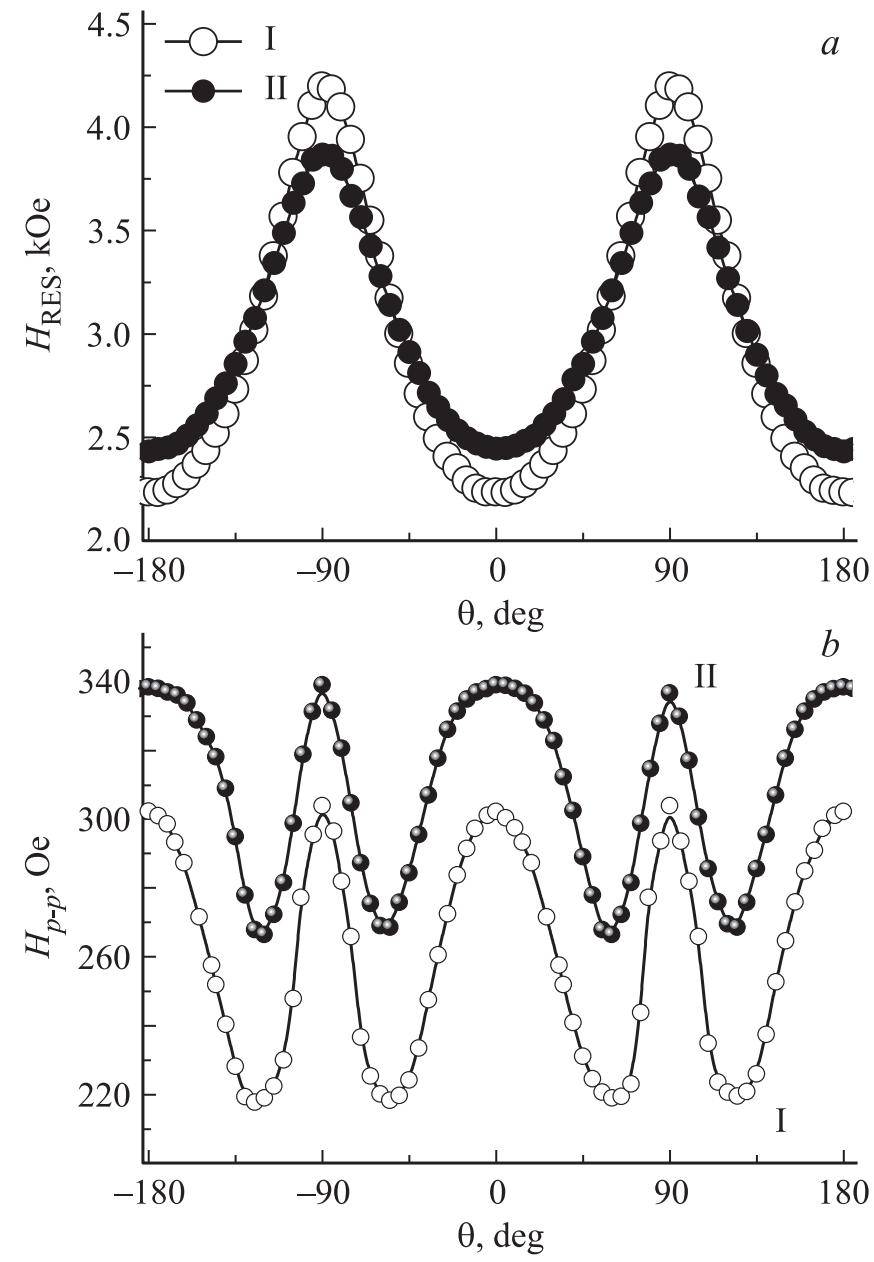

Рис. 2. Угловые зависимости резонансного поля $H_{\mathrm{RES}}(a)$ и ширины линии $H_{\text {Р-P }}(b)$ для монослоя (I) и бислоя (II).

толстом слое вентиля толщиной $1.1 \mathrm{~nm}$ эти параметры принимали следующие значения: $M_{S}=768 \mathrm{G}$, $K_{2 \perp}=4.05 \cdot 10^{6} \mathrm{erg} / \mathrm{cm}^{3}, \quad K_{S 2}=0.4 \mathrm{erg} / \mathrm{cm}^{2}$. Отметим, что, несмотря на одинаковую толщину слоев $1.1 \mathrm{~nm}$ в монослойном и бислойном образцах, параметры магнитной анизотропии в них различались. Монослой проявлял бо́льшую склонность к перпендикулярной анизотропии.

Ширина линии как правило определяется суммой вкладов трех компонент: уширения $\Delta H_{G}$ вследствие конечного времени затухания спиновой прецессии (определяется параметром Гильберта $G)$, уширения $\Delta H_{2 \mathrm{mag}}$ вследствие двухмагнонного рассеяния и неоднородного уширения линии $\Delta H_{\text {inh }}$ [16]. В тонких кристаллических пленках высокой однородности с большими значениями намагниченности влиянием неоднородного уширения $\Delta H_{\text {inh }}$ можно пренебречь, в то время как величины $\Delta H_{G}$ и $\Delta H_{2 \mathrm{mag}}$ сопоставимы. В соответствии с данными работы [16] $\Delta H_{G}=2 \alpha(\omega / \gamma)$. Таким образом, ширина резонансной линии может быть записана в виде $\Delta H=\Delta H_{G}+\Delta H_{2 \operatorname{mag}} \sim 2 \Delta H_{G}=4 \alpha(\omega / \gamma)$, откуда параметр Гильберта может быть оценен как $\alpha=(1 / 4) \Delta H /(\omega / \gamma)$. В нашем случае $\omega / \gamma=3358 \mathrm{Oe}$, 


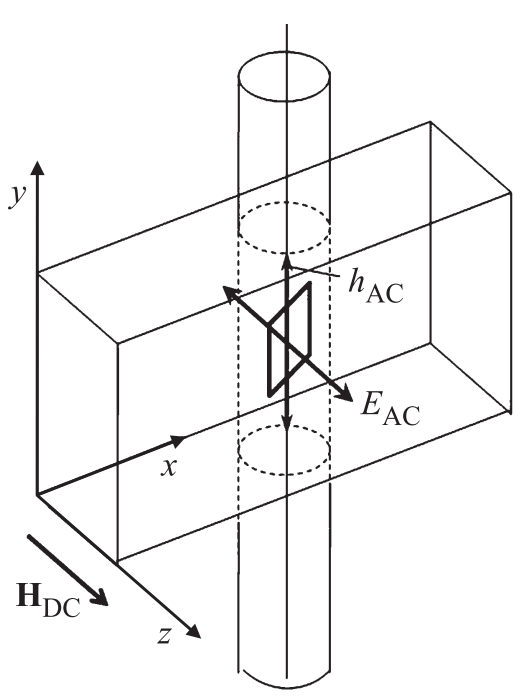

Рис. 3. Схема измерений ФМР в резонаторе $H_{102}$. Магнитная компонента микроволнового поля осциллирует в плоскости $x y$, электрическая компонента - вдоль оси $z$.

$\Delta H \sim 200-300$ Ое. Отсюда $\alpha \sim 0.015$ для монослоя и $\alpha \sim 0.022$ для бислоя. Полученные значения параметра Гильберта лежат внутри диапазона 0.004-0.027, допустимого для эпитаксиальных структур CoFeB с ГMC, применяемых в спинтронике.

Обсудим теперь форму линий ФМР. Асимметрия линий ФМР также часто бывает связана с потерями, возникающими в скин-слое в результате диссипации электрической компоненты микроволнового поля, проходящего через образец (линия Дайсона) [17]. Схема, показывающая направление электрической и магнитной компонент микроволнового поля относительно нормали к плоскости образца, для произвольного угла $\theta$ между направлением постоянного магнитного поля $H_{\mathrm{DC}}$ и направлением нормали к плоскости образца представлена на рис. 3. Вдоль оси $x$ в резонаторе укладываются две полуволны $h_{\mathrm{AC}}$. Образец находится в пучности колебаний микроволнового поля $h_{\mathrm{AC}}$, поэтому в области нахождения образца магнитная компонента микроволнового поля осциллирует вдоль оси $y$.

Электрическая компонента микроволнового поля осциллирует вдоль оси $z$ (вдоль направления постоянного магнитного поля спектрометра). Наибольшая величина диссипации электрической компоненты микроволнового поля достигается при $\theta=90^{\circ}$, когда вектор электрической компоненты направлен вдоль плоскости образца. При $\theta=0^{\circ}$ колебания электрической компоненты происходят в направлении, перпендикулярном плоскости образца, и потери энергии микроволнового поля минимальны. Таким образом, если бы асимметрия линий ФМР была обусловлена только наличием диэлектрических потерь, то при заданной величине угла $\theta$ отношение амплитуд левого $A_{1}$ и правого $A_{2}$ крыльев линии должно быть одинаковым как для монослоя, так и для бислоя, а наиболее сильное различие амплитуд $A_{1}$ и $A_{2}$ должно было бы достигаться при $\theta=90^{\circ}$. Однако, как видно из рис. 1, в нашем случае это не так. Для монослойного образца линия симметрична на всем диапазоне углов $\theta$ от 0 до $90^{\circ}$, в то время как для бислоя линия ФМР асимметрична, наибольшее различие амплитуд $A_{1}$ и $A_{2}$ достигается при $\theta=60^{\circ}$. Это означает, что асимметричность линий ФМР для бислоя в нашем случае не может быть объяснена в рамках модели Дайсона и, скорее всего, является следствием наложения двух линий, по одной от каждого ферромагнитного слоя. Разложение линии на две лоренцевы компоненты и анализ констант анизотропии в этих условиях показали, что значения констант анизотропии в пределах погрешности получаются равными приведенным выше для случая, когда спектр анализировался как одиночная линия.

Нельзя исключить также и взаимного влияния двух близко расположенных ферромагнитных слоев $\mathrm{CoFeB}$ в спиновом вентиле. Толщина прослойки Та между ними обеспечивает обменное взаимодействие между слоями и таким образом изменяет эффективную толщину слоев, что также может приводить к изменению констант анизотропии и фактора затухания прецессии. Отметим, что при комнатной температуре перемагничивание слоев в магнитном поле спектрометра, развертка которого занимает 10-15 min, сопровождается релаксационными процессами, связанными с доменными стенками и зародышами обратной намагниченности. Эти объекты создают предпосылки неоднородной намагниченности пленок и способны увеличивать значение константы демпфирования, а также приводить к асимметрии линии ФМР.

\section{4. Заключение}

Обнаружены различия констант анизотропии и фактора затухания спиновой прецессии в пленке $\mathrm{MgO} / \mathrm{CoFeB} / \mathrm{MgO} / \mathrm{Ta}$, содержащей один ферромагнитный слой $\mathrm{CoFeB}$, и в спиновом вентиле $\mathrm{MgO} / \mathrm{CoFeB} / \mathrm{Ta} / \mathrm{CoFeB} / \mathrm{MgO} / \mathrm{Ta}$, состоящем из двух ферромагнитных слоев $\mathrm{CoFeB}$, разделенных антиферромагнитным слоем Та, один из слоев которого имеет такую же толщину, как и пленка в однослойном образце. Эти различия объясняются разной удельной долей интерфейca $\mathrm{CoFeB} / \mathrm{MgO}$, обеспечивающего перпендикулярную анизотропию путем гибридизации $3 d$-орбиталей переходных металлов $(\mathrm{Co}, \mathrm{Fe})$ с $\mathrm{O}_{p}^{2}$-орбиталями оксида $\mathrm{MgO}$.

\section{Список литературы}

[1] S. Ikeda, K. Miura, H. Yamamoto, K. Mizunuma, H.D. Gan, M. Endo, S. Kanai, J. Hayakawa, F. Matsukura, H. Ohno. Nature Mater. 9, 721 (2010).

[2] X. Wang. Metallic spintronic devices. CRC Press, Fl., USA (2014). 273 p.

[3] L. Cuchet, B. Rodmacq, S. Auffret, R.C. Sousa, I.L. Prejbeanu, B. Dieny. Sci. Rep. 6, 21246 (2016). 
[4] T. Zhu, P. Chen, Q.H. Zhang, R.C. Yu, B.G. Liu. Appl. Phys. Lett. 104, 202404 (2014).

[5] R. Soucaille, M. Belmeguenai, J. Torrejon, J.V. Kim, T. Devolder, Y. Roussigne, S.M. Cherif, A.A. Stashkevich, M. Hayashi, J.P. Adam. Phys. Rev. B 94, 104431 (2016).

[6] N. Lei, T. Devolder, G. Agnus, P. Aubert, L. Daniel, J.V. Kim, W. Zhao, T. Trypiniotis, R.P. Cowburn, C. Chappert, D. Ravelosona, P. Lecoeur. Nature Commun. 4, 1378 (2013).

[7] S.M. Watson, T. Hauet, J.A. Borchers, S. Mangin, E.E. Fullerton. Appl. Phys. Lett. 92, 202507 (2008).

[8] S. Mangin, L. Thomas, F. Montaigne, W. Lin, T. Hauet, Y. Henry. Phys. Rev. B 80, 224424 (2009).

[9] H. Yang, M. Chshiev, B. Dieny, J. Lee, A. Manchon, K. Shin. Phys. Rev. B 84, 054401 (2011).

[10] D.B. Gopman, D. Bedau, S. Mangin, E.E. Fullerton, J.A. Katine, A.D. Kent. J. Appl. Phys. 115, 113910 (2014).

[11] S. Mangin, A. Sulpice, G. Marchal, C. Bellouard, W. Wernsdorfer, B. Barbara. Phys. Rev. B 60, 1204 (1999).

[12] S.A. Oliver, C. Vittoria, E. Schloemann, H.J. Van Hook, R.W. Tustison. J. Appl. Phys. 63, 3802 (1988).

[13] B. Schulz, K. Baberschke. Phys. Rev. B 50, 13467 (1994).

[14] G. Gubbiotti, G. Carlotti, B. Hillebrands. J. Phys.: Condens. Matter 10, 2171 (1998)

[15] L. Baselgia, M. Warden, F. Waldner, S.L. Hutton, J.E. Drumheller, Y.Q. He, P.E. Wigen, M. Marysko. Phys. Rev. B 38, 2237 (1988).

[16] J. Dubowik, K. Załeski, H. Głowinski, I. Goscianska. Phys. Rev. B 84, 184438 (2011).

[17] Ч. Пул. Техника ЭПР-спектроскопии. Мир, М. (1970). C. 448. 